\title{
Mobile breathers in a Nonlinear model for DNA breathing
}

\author{
Hernán Cortez Gutiérrez $^{1+}$, Elso Drigo Filho ${ }^{2}$, José Roberto Ruggiero ${ }^{2}$, Milton Cortez Gutierrez ${ }^{3}$ \\ ${ }^{1}$ Universidad Nacional del Callao, Facultad de Ciencias de la Salud, 306 Juan Pablo II Av, Callao, Bellavista, 07001, Perú \\ ${ }^{2}$ São Paulo State University (Unesp), Institute of Biosciences, Languages, and Exact Sciences (Ibilce), 2265 Cristóvão Colombo St, São José Rio \\ Preto, São Paulo, 15054-000, Brazil \\ ${ }^{3}$ Universidad Nacional de Trujillo, Departamento de Matemática, Juan Pablo II Av, Trujillo, Peru \\ + Corresponding author: Hernan Cortez Gutierrez, e-mail address: hcortez@unac.pe
}

\section{ARTICLE INFO}

Article history:

Received: August 27, 2017

Accepted: December 28, 2017

Published: December 30, 2017

\author{
Keywords: \\ 1. harmonic bifurcation \\ 2. mobile breather \\ 3. center of energy \\ 4. inhomogeneity \\ 5. DNA breathing
}

ABSTRACT: Objectives. Analyze the DNA dynamics in PeyrardBishop-Dauxois model (PBD) with different control parameters using its energy center of the mobile "breather". Materials and methods. We used the Peyrard-Bishop-Dauxois mathematical model and the MATLAB software for studying the DNA dynamic using Morse potential, Symmetric Morse and the "hump" potential for simulating the interactions which arise the pile up. Results. It has been observed that the analytical and computational methods allow to detect the influence of the potentials of the PBD model in the behavior of the energy center in the presence of a couple of base $\mathrm{A}$ (adenine) or $\mathrm{T}$ (thymine) using the control of parameter $\alpha=-0.30$ and velocity of mobile breather: $\mathrm{v}_{0}=0.1$. In the case of Morse potential, the center of energy respect to the mobile breather undergoes a change in its trajectory and produce a DNA breathing. Conclusions. Analytical and computational approaches can be used for obtaining differences respect to the DNA dynamics using different control parameters: velocity of BM and inhomogeneity. The potential "hump" may decrease the reflective effect with the indicated parameters to the effect on the energy center to the mobile breather.

\section{Introduction}

DNA is an important basis of molecular biology ${ }^{1}$. The dynamic in DNA depends on the physical parameters that modify the behavior of the base pairs and show large amplitude fluctuations known as the "breathing" of DNA $^{2}$. We have some references about this dynamics in the model of Peyrard and Bishop ${ }^{3-14}$. This model exhibits spatially localized oscillations denoted as "discrete breathers" . Flach, A. Gorbach ${ }^{16}$ give the theory of the discrete breathers (DB) and applications.

Forinash, Peyrard and Malomed ${ }^{17}$ proved the influence of an impurity in the movement of discrete breathers in Klein-Gordon (KG) chains. The method used for calculating discrete breathers in $(\mathrm{KG})$ lattices consists on approximating the dynamical equations by a Nonlinear
Schrodinger Equation (NLS) and moving the breather by an envelope solution ${ }^{17}$.

Cuevas, Palmero, Romero and Archilla have found that the moving breather can cross the impurity, and can be reflected by it, or can be trapped ${ }^{18,19}$.

The problem is to determine the parameters of the model to generate a trapped breather (TB) which is very important for the DNA transcription. The results obtained in our simulation verify the existence of breather trapped with the conditions described by Cuevas, Palmero and Archilla $^{18}$. The trapped breather (TB) depends on the impurity and the initial velocity of mobile breather.

In addition, we have also observed that the PBD model with "hump" Morse potential and inappropriate initial velocity have different vibration. 
We conjecture that "hump" Morse potential with appropriate initial velocity of mobile breather and impurity can generate a trapped breather. The results of the simulations can be applied to study of moving breather collisions in the PBD model (for a review, $\mathrm{see}^{20}$ ).

\section{Experimental}

2.1. The PBD model with modified Morse potentials and the inhomogeneity for the Morse potential

At a first stage, the dynamics of this PBD model is analyzed by the equations:

$V$ : Potential function

$K$ : Coupling constant

velocity $=u ; u=$ stretching of the hydrogen bonds

$N=$ Number of the pairs of basis.

$H=\sum_{n=1}^{N} \frac{m}{2} u_{n}^{2}+\sum_{n=1}^{N} \frac{K}{2}\left(u_{n}-u_{n-1}\right)^{2}+\sum_{n=1}^{N} V_{n}\left(u_{n}\right)$

The associate equations for (4) are a system of equations $(n=1,2 \ldots N)$

$\ddot{u}_{n}+V^{\prime}\left(u_{n}\right)+K\left(2 u_{n}-u_{n-1}-u_{n+1}\right)=0$,

( $V^{\prime}\left(u_{n}\right)$ being the derivative of the potential)

We consider potentials of the form:

$$
V_{n}\left(x_{n}-y_{n}\right)=D_{n}\left[V\left(x_{n}-y_{n}\right)\right]
$$

$V$ is the potential and $D_{\text {n }}$ represents a discontinuity in the bases. In case of a discontinuity on the site only $n_{0}, D_{\mathrm{n}}$ is the form

$$
D_{n}=D_{0}\left(1+\alpha \delta_{n, n 0}\right),
$$

and the parameter $\alpha$ is the non-homogeneity (magnitude of the impurity) and considered in the interval $[-1, \infty)$ and $D_{0}$ a constant.

In the case of the Potential of Morse are:

$$
V_{n}\left(x_{n}-y_{n}\right)=D_{n}\left\{\exp \left[-a_{n}\left(x_{n}-y_{n}\right)\right]-1\right\}^{2},(8)
$$

$D_{\mathrm{n}}$ is the depth of potential and $a_{\mathrm{n}}$ represents the amplitude of the potential of Morse of the site $n$.

We consider the potentials: (symmetric Morse potential)

$V(u)=\frac{1}{2}[\exp (-|u|)-1]^{2}$

("hump" Morse potential)

$V(u)=0.15806[\exp (-4 u)-1]^{2}$, if $\mathrm{u}<0$

$V(u)=2.52 u^{2}+-4.05 u^{3}+1.70 u^{4}$, if $0<\mathrm{u}<1$

$V(u)=0.0857+4[\exp (-4 u)](u+1 / 4)$, if $\mathrm{u}>1$.

\subsection{Numerical solutions of the equations (5)}

Using the approximation for the oscillator $n$ and $T=2 \pi / \mathrm{w}_{\mathrm{b}}$

$u_{n}=z_{n}^{0}+\sum_{k=1}^{k_{m}} 2 z_{n}^{k} \cos \left(k \omega_{b} t\right)$.

and substituting in (5) one has

$$
k^{2} \omega_{b}^{2} z_{n}^{2}+V_{n}^{\prime k}+\mu\left(2 z_{n}^{k}-z_{n+1}^{k}-z_{n-1}^{k}\right)=0,
$$

which depend on the parameter $K$, and $V_{n}^{\prime}{ }^{k}$ is the $k^{\text {th }}$ Fourier coefficient for the periodic function $V^{\prime}\left(u_{\mathrm{n}}(\mathrm{t})\right)$.

In Figure 1 is depicted the example for the potential (equation 9) the solution of equations (5) denominated breather.

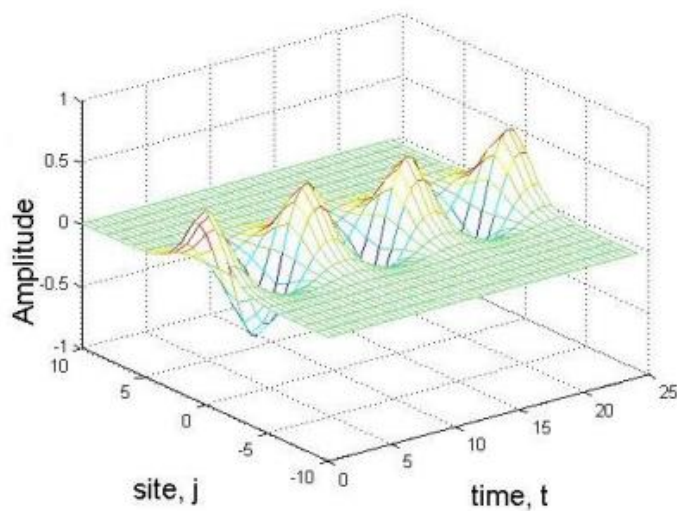

Figure 1. The solution of equations (5) for the case of potential (equation 9) with $K=0.3$ and $w b=0.8$.

Remark 1 The dynamics of the DNA is a set of coupled oscillators, and the vibrational motion is equivalent to equations (5) which depend on the several control parameters: potential modified of Morse and constant $K$ of coupling. Parametric changes in the potentials imply changes in the amplitudes of the vibrations. For example, for the potential (equation 9), 
the maximum amplitude of the vibrations is 0.6. In biological terms, this change can be the effect of a disease or pharmaceutical control treatment.

\subsection{STABILITY: Harmonic bifurcation}

We analyze the stability of the breather solution. Let us introduce a function $\tilde{u}_{\mathrm{n}}(\mathrm{t})=u_{\mathrm{n}}(\mathrm{t})+\varepsilon_{\mathrm{n}}(\mathrm{t})$, where $u_{\mathrm{n}}(\mathrm{t})$ is the periodic breather solution shown in Figure 1 and $\varepsilon_{\mathrm{n}}(\mathrm{t})$ is a perturbation: $\tilde{u}_{\mathrm{n}}(\mathrm{t})$ must satisfy the system (equations 5) and expanding around $u_{\mathrm{n}}(\mathrm{t})$ to first order (linearization), we obtain the following system of equations for $\varepsilon_{\mathrm{n}}(\mathrm{t})$

$$
\ddot{\varepsilon}_{n}+\left(V^{\prime \prime}\left(u_{n}(t)\right)\right) \varepsilon_{n}+K\left(2 \varepsilon_{n}-\varepsilon_{n-1}-\varepsilon_{n+1}\right)=0
$$

We can associate a monodromy matrix for this equation with Floquet multipliers ${ }^{7}$.

Remark 2 The solution is stable if the modules of Floquet multipliers are one. The especial instability ("harmonic bifurcation") in our case happens when a pair of Floquet multipliers merges at $\lambda=1$ and splits off circle onto the positive real axis, in Figure 2.

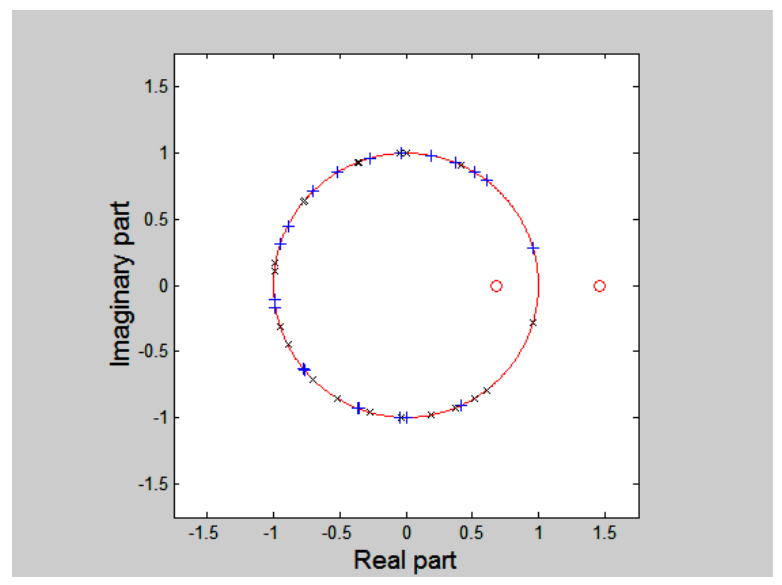

Figure 2. The instability "harmonic bifurcation". Case PBD Model with $K=0.13$.

\subsection{Breather mobile and breather trapped}

Once the system of equations (5) is worked out by Runge Kutta method and has seen its stability till the parameter $K=0.13$, one construct in breather mobile considering the initial conditions of the position and average speed of each position " $n$ " respect to the harmonic oscillation corresponding to the DNA and one get construct its corresponding center of energy of the breather mobile ${ }^{13}$ (see Figure 3).

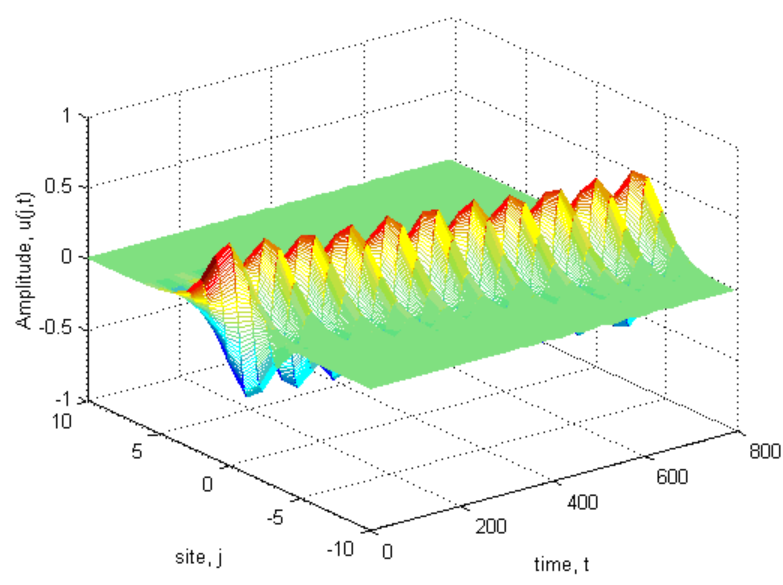

Figure 3. Breather mobile for the Symmetric Morse potential.

Remark 3 The maximum amplitude of the breather mobile has 0.6 angstrom for the symmetric Morse potential. by

The center of energy of the breather mobile is given

$$
X_{E}=\sum_{n=1}^{N} n H_{n}^{d} / H
$$

where the density energy is the form

$$
H_{n}^{d}=\frac{1}{2} \dot{u}_{n}^{2}+\frac{K}{4}\left(u_{n}-u_{n-1}\right)^{2}+\frac{K}{4}\left(u_{n+1}-u_{n}\right)^{2}+V_{n}\left(\sqrt{2} u_{n}\right)
$$

For the case of Morse potential the breather trapped is obtained with the additional condition: $\alpha, \varepsilon(-0.49$, $0.02)$ and the initial velocity of $B M: v_{0}=0.1^{18}$.

It is very important the initial velocity of the BM for the displacement a long of sites of DNA and can be produced of DNA breathing.

This couple of parameters $\left(\alpha, \mathrm{v}_{0}\right)$ is transcendental for DNA breathing.

\subsection{Test of simulation for the evolution of the center of energy with modified Morse potential and Morse potential}

The dynamic respect to the DNA with $N=251$ a couple of bases is watched more precisely analyzing its center of energy for two types of potential considering an impurity in the position $n_{0}=26$ and with the control parameter $\alpha=-0.30, D_{\mathrm{n}}=0.5\left(n \neq n_{0)}\right.$. The interaction respect to the center of energy for two types of potential between the model of Morse and the model "hump" Morse is showed in the Figure 4. 


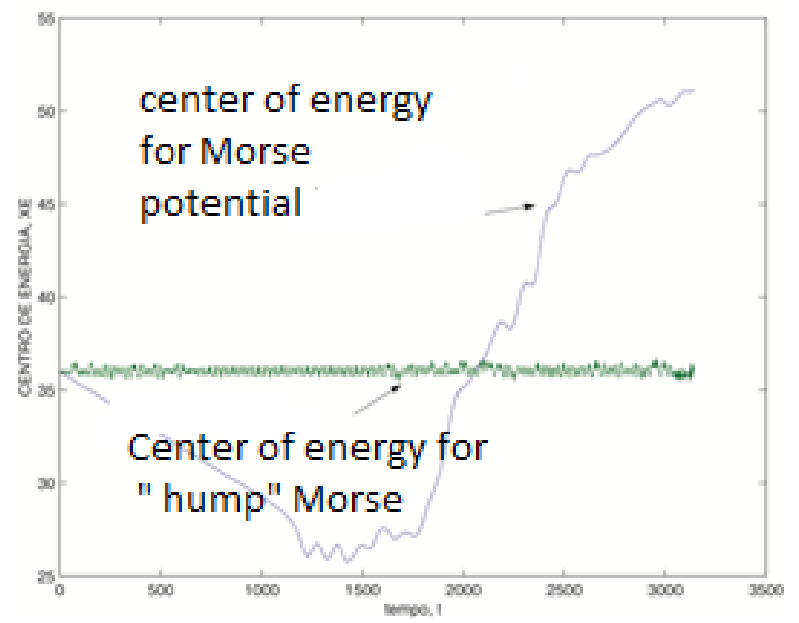

Figure 4. Evolution of the center of energy considering two types of Morse potentials with the parameters: $N=251, K=0.13$ and the position of the inhomogeneity $n_{0}=26$ for the Morse potential.

Remark 4 In the reference ${ }^{13}$ we have the complete study of the Figure 4 considering the sequence of Insulin with $N=251$ pairs of basis. In Figure 4 we have the trapped case for the interaction of the center of energy when we consider the Morse potential and the position of the inhomogeneity.

Remark 5 In Figure 4 we do not have the reflected case for the interaction of the center of energy when we consider the "hump" Morse potential.

\section{Discussion}

In the Remark 3: The maximum amplitude of the breather mobile has 0.6 angstrom for the symmetric Morse potential. In this case is interesting to simulate interactions of the center of energy with inhomogeneity. We have obtained an appropriate center of energy for the initial velocity of $0.1^{12}$.

In the Remark 4: We have shown the center of energy is trapped with the control in the inhomogeneity $\boldsymbol{n}_{\mathbf{0}}=\mathbf{2 6}$ for the Morse potential. This case is favorable for the DNA transcription because we need the breather trapping $^{18}$.

In the Remark 5: We have shown the center of energy is not trapped for the "hump" Morse potential. This case is not favorable for the DNA transcription because we need the breather trapping ${ }^{18}$. In this case is necessary to introduce the control parameter appropriate of inhomogeneity for obtaining a breather trapping using the center of energy ${ }^{18}$. Another control parameter is the initial velocity of the breather mobile as showed in reference ${ }^{16}$. For Figure 4, it is firstly necessary an appropriate initial velocity for the simulations of the center of energy in interactions with inhomogeneity (for a review, see ${ }^{12}$ and Figure 5).

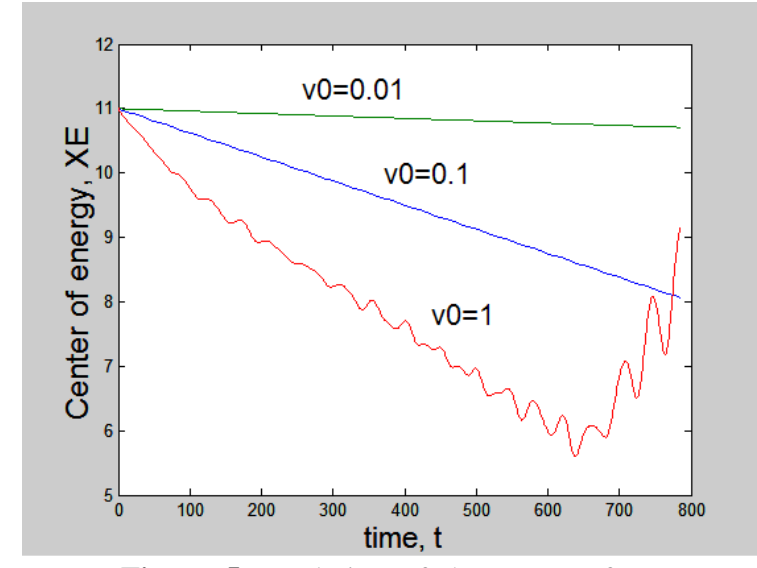

Figure 5. Evolution of the center of energy considering of symmetric Morse potential. with the velocities: $0.01,0.1$ and 1 .

Our results are similar as obtained the reference ${ }^{18}$ with the control of parameters for the BT (Breather trapped):

1. Initial velocity for the BM with the parameter $\lambda=0.1$

2. Inhomogeneity $\alpha=-0.30$ for the Morse potential in the PBD model.

The proposed method can be applied to verify the breather rebounds when the parameters $\alpha, \varepsilon(-0.54$, $0.49)^{18}$ and using another initial velocity for mobile breather.

Even though, a reflection is observed after a short period of time in figure 4 . In fact, many breathers trapped collide and facilitate the transcript process (for a review, see $^{20}$ ).

The theory of control optimum can be used for explore the effective breather trapped.

Finally the breather trapped act as a precursor of the transcription bubble of DNA ${ }^{21}$. These BTs can interact with another breathers and collect energy ${ }^{22}$.

\section{Conclusions}

This work has proposed state influence in the dynamic of DNA using the potentials, initial velocity of the breather mobile and the inhomogeneity as parameters of control. Next we have obtained different amplitudes of the breather solution depending of the potential. Finally, it is very important to emphasize the importance of the analytical and computational methods in the study of the components of DNA.

Next, the interaction of the breather mobile with the inhomogeneity of the DNA has been proposed for performing changes in the trajectory of the center of energy for the Morse potential with implications in the transcription process of DNA. 


\section{References}

[1] Micklos. D., DNA Science. Laboratory Press. 2003.

[2] James, G., Continuation of discrete breathers from infinity in a nonlinear model for DNA breathing. Applicable Analysis, 2009.

[3] Peyrard, M., Bishop, A. R., Statistical Mechanics of a nonlinear model for DNA, Physica Review Letters 62 (1989) $2755-2758$

https://doi.org/10.1103/PhysRevLett.62.2755

[4] Cuevas, J., Localización y Transferencia de Energia en Redes Anarmónicas No Homogéneas, Ph. D. Thesis, Universidad de Sevilla, Sevilla, España, 2003.

[5] Mackay, R. S., Aubry, S., Proof of existence of breathers for time-reversible for Hamiltonian networks of weakly coupled oscillators, Nonlinearity 7 (1994) 1623 1643.

[6] Jefferson, J., Structural dynamics in DNA damage signaling and repair, Curr. Opin. Struct. Biol. 20(3) (2010) 283-294. https://doi.org/10.1016/j.sbi.2010.03.012

[7] Hoppensteadt, F. C., Analysis and Simulation of Chaotic Systems, Spring-Verlag, New York, 2000.

[8] Chen, D., Aubry, S., Tsironis, G. P., Breather mobility in discrete lattices, Physical Review Letters $77 \quad$ (1996) 4776-4779. https://doi.org/10.1103/PhysRevLett.77.4776

[9] Cuevas, J., Palmero, F., Archilla, J. F. R., Romero, F. R., Moving discrete breather in a Klein-Gordon chain with an impurity, J. Phys. A, 35 (2002) 10519-10530.

[10] Aubry, S., Cretegny, T., Mobility and Reactivity of Discrete Breathers, Physica D, 119 (1998) 34-46. https://doi.org/10.1016/S0167-2789(98)00062-1

[11] Howard, J., Linear stability of natural sympletic maps, Physics Letters A 246 (1998) 273-283. https://doi.org/10.1016/S0375-9601(98)00507-6
[12] Cortez, H., Drigo Filho, E., Ruggiero, J. R., Breather stability in one dimensional Lattices with a symmetric Morse Potential, TEMA. Tend. Mat. Appl. Comput. 9(2) 205-212. https://doi.org/10.5540/tema.2008.09.02.0205

[13] Cortez, H., Tese de doutorado. Modelo Dinâmico e estatístico aplicado à transição de fase. UNESP. 2009

[14] Gutiérrez, H.C., Drigo Filho, E., Ruggiero, J.R., Gutierrez, M.C., Fuentes Rivera, L.V., Thermodynamics of DNA with “ hump” Morse potential, Eclética Quimica 41 (2016) 60-65. https://doi.org/10.26850/16784618eqj.v41.1.2016.p60-65

[15] Peyrard, M., Nonlinear dynamics and statistical physics of DNA, Nonlinearity 17(2) (2004) R1-R40.

[16] Flach, S. Gorbach, A. V., Discrete breathersAdvance in theory and applications, Physics Reports 467 (1-3) (2008) 1-116. https://doi.org/10.1016/j.physrep.2008.05.002

[17] Forinash, K., Peyrard M., Interaction of discrete with impurity modes, Physical Review E 49 (4) (1994) 3400-3411. PMID:9961608

[18] Cuevas, J., Palmero, F., Archilla, J. F. R., Moving discrete breathers in a Klein-Gordon chain with an impurity, Journal of Physics 35(49) (2002) 10519-10530.

[19] Alvarez, A., Romero, F., Breather trapping and breather transmission in a DNA model with an interface, Eur. Phys. J. B51 (2006) 119-130. https://doi.org/10.1140/epjb/e2006-00191-0

[20] Alvarez. A., Moving Breathers collisions in the Peyrard Bishop DNA model. International Conference on Complex Science, 2009.

[21] Thing, J., Peyrard, M., Effective breather trapping mechanism for DNA transcription, Physics Rev. 53(1) (1996) 1011-1020. PMID: 9964336

[22] Bang, O., Peyrard, M., High order breather solutions to a discrete nonlinear Klein-Gordon model, Physica D 81(1-2) (1995) 9-22. https://doi.org/10.1016/01672789(94)00202-2 